\title{
Management of the Primary Tumor and Limited Metastases in Patients With Metastatic Pancreatic Cancer
}

\author{
Joseph M. Herman, MD, MSc ${ }^{\mathrm{a}}$; John P. Hoffman, MD ; Sarah P. Thayer, MD, PhD'; and Robert A. Wolff, MD
}

\begin{abstract}
New combinations of cytotoxic chemotherapy have been proven to increase response rates and survival times compared with single-agent gemcitabine for patients with metastatic pancreatic cancer. These responses have been dramatic for a subset of patients, therefore raising questions about the management of limited metastatic disease with surgery or other ablative methods. Similarly, for patients having a complete radiographic response to chemotherapy in the metastatic compartment, whether to consider local therapy in the form of radiation or surgery for the primary tumor is now an appropriate question. Therefore, collaboration among experts in surgery, medical oncology, and radiation oncology has led to the development of guiding principles for local therapies to the primary intact pancreatic tumor for patients with limited metastatic disease and those who have had a significant response after systemic therapy. (J Natl Compr Canc Netw 2015;13:e29-e36)
\end{abstract}

Pancreatic cancer (PCA) is one of the most challenging malignancies faced by patients, their families, and their physicians. Symptoms related to metastatic disease (eg, malaise, fatigue, cachexia, and venous thromboembolism) may all contribute to poor quality of life. Moreover, the local component of PCA may also be associated with significant morbidity, including pain, biliary obstruction,

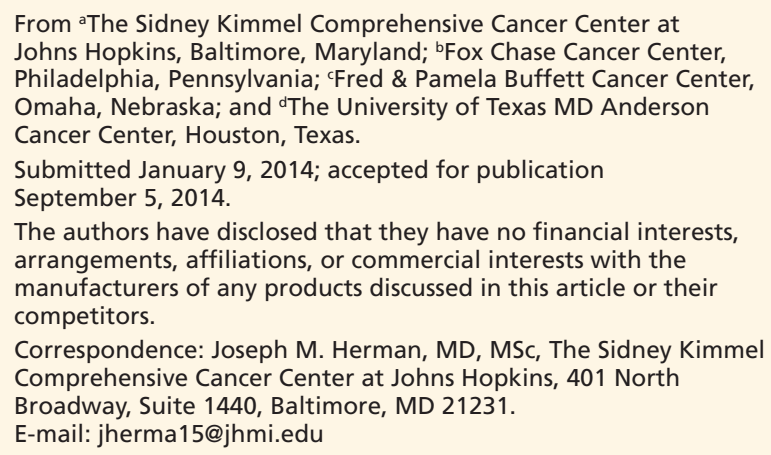

gastrointestinal dysfunction or bleeding, and exocrine or endocrine pancreatic insufficiency. Fortunately, multidisciplinary engagement and multiple therapeutic modalities have gradually improved and been refined to optimize survival while preserving quality of life.

Currently, a convergence of several improved techniques and technologies, coupled with a better understanding of underlying tumor biology, now enables physicians to consider more aggressive therapies for select patients who present with limited metastatic disease.

First, improvements in cross-sectional imaging have facilitated the detection of very low-volume metastatic disease. Such metastatic foci, which can be limited to 1 or 2 lesions in a single organ, may have gone undetected in previous years. In addition, laparoscopy seems to be particularly effective in identifying occult metastatic disease that may otherwise be undetected by modern imaging modalities., ${ }^{1,2}$

Second, a broader array of radiation options that include image-guided (IGRT) and stereotactic body radiation therapy (SBRT) have demonstrated encouraging efficacy in the treatment of primary and metastatic tumors while minimizing toxicity. Importantly, these techniques can be delivered over relatively short time courses, thus limiting the delay of systemic therapies. However, longer follow-up is needed to determine the full extent of late toxicity.

Third, recent advances in combination chemotherapy yield higher response rates and survival. Although uncommon, some isolated case reports of complete or near-complete radiographic responses have been observed after combination chemotherapy regimens. With the widespread adoption of novel combination chemotherapy regimens, larger subsets of 
Herman et al

patients with metastatic disease may be considered for novel clinical trials to manage the metastatic compartment as a way to preserve or improve quality of life.

Fourth, image-guided percutaneous radiofrequency ablation (RFA) and laparoscopically assisted RFA may allow for management of limited metastatic disease and spare patients the toxicity associated with ongoing cytotoxic chemotherapy.

Lastly, the morbidity and mortality associated with metastatectomies or primary PCA resections continues to decline, particularly in high-volume centers. Whether these interventions lead to improved overall survival (OS) or disease-free survival (DFS) in the setting of limited metastases is not yet known.

\section{Role of Chemotherapy in Metastatic PCA}

In 1996, weekly doses of gemcitabine proved to be modestly superior to weekly bolus fluorouracil. Furthermore, clinical benefit, an end point not commonly used in subsequent trials, was improved using gemcitabine compared with fluorouracil. ${ }^{3}$

Unfortunately, during the subsequent 15 years, although cytotoxic gemcitabine doublets often led to slightly higher objective response rates, no clinically or statistically significant survival benefit was observed in large randomized trials in which gemcitabine alone was compared with various gemcitabine doublets (cisplatin, oxaliplatin, irinotecan, fluorouracil, and capecitabine). The FDA approved the addition of erlotinib to gemcitabine, but the benefit was a mere few-week improvement in median OS. Moreover, the combination of gemcitabine with a variety of molecular agents - specifically bevacizumab, cetuximab, tipifarnib, and axitinib-demonstrated no improvement in objective responses or survival. ${ }^{4}$

In 2011, European investigators demonstrated that a combination of folinic acid, fluorouracil, irinotecan, and oxaliplatin (FOLFIRINOX) led to significantly higher response rates and survival times compared with gemcitabine (response rate, $32 \%$ vs 9\%; median OS, 11.1 vs 6.8 months, respectively) in a group of patients with metastatic PCA. ${ }^{5}$ More recently, the combination of gemcitabine and nabpaclitaxel has likewise proven to be superior to gemcitabine alone for patients with metastatic PCA. ${ }^{6}$ Of interest, the experimental arm generated complete radiographic responses in both trials, an observation not seen in patients treated with gemcitabine alone. However, dramatic tumor responses are not limited to FOLFIRINOX or gemcitabine plus nab-paclitaxel, and clinically significant anticancer activity has been reported for combinations of gemcitabine, docetaxel, and capecitabine (GTX).

Although progress in the treatment of PCA has been incremental and uneven, as the scientific revolution in genomics, proteomics, and metabolomics converges with a greater understanding of host response and tumor microenvironment, more rapid advances in systemic therapy for PCA are certain to emerge. This may eventually lead to curative systemic therapies for patients with advanced disease. In the near-term, however, given the improved response rates currently being seen in clinical trials and practice, integration of other anticancer modalities can now be contemplated in select patients with a limited metastatic burden of disease.

\section{Role of Surgical Management in Metastatic PCA}

A small number of studies have identified that surgery can have a statistically significant survival advantage in selected patients with minimal metastatic disease. ${ }^{8}$ However, because the survival difference is so small, the clinical benefit may be negligible when considering a 6 - to 8 -week postoperative recovery time and a $35 \%$ to $45 \%$ operative morbidity. Thus, surgery in the presence of metastatic disease has been discouraged.

Conversely, the present rationale for proposing resection of the primary tumor in patients with metastatic PCA has been fueled by subset analyses that reveal significant improvements in survival of more than 4 months in patients who received both chemotherapy and surgery.,10 A similar survival effect (3 months) was seen in patients who underwent palliative pancreaticoduodenectomy with gross residual disease. ${ }^{11,12}$ These findings, in addition to more effective multimodality therapies, suggest that a select group of patients with low-volume metastatic disease and good performance status may benefit from resection of the primary tumor if they also receive aggressive chemotherapy. However, other studies have shown no survival benefit when patients with meta- 
static disease undergo aggressive surgical resection of the primary tumor and metastases. ${ }^{13}$ Therefore, additional clinical trials are warranted before these recommendations may be applied to the general population, because the subset of healthier patients selected for resection reflects potential selection bias.

Advances in radiologic and molecular diagnostic techniques have improved the ability to detect signs of minimal metastatic disease in patients with earlystage PCA. If all surgical candidates are assumed to have microscopic metastatic disease, how is it possible to properly select which patients should undergo upfront surgery versus systemic chemotherapy? Or should all surgical patients receive some form of neoadjuvant therapy? The following sections discuss these issues, as potential prognostic and predictive biomarkers in PCA are explained.

\section{Management of Positive Peritoneal Cytology}

The AJCC and NCCN consider positive peritoneal cytology (PPC) as M1, metastatic disease. ${ }^{14} \mathrm{PPC}$ is often associated with advanced-stage disease and has therefore been correlated with a poor prognosis and considered a contraindication to resection. ${ }^{15,16}$ However, many of these earlier studies did not have a large population of resected patients with PPC. Therefore, a fair amount of controversy remains regarding PPC in patients with potentially resectable PCA with no other evidence of distant metastasis. Recently, several large patient series examining the clinical implication of PPC in resectable PCA revealed no difference in OS after resection. ${ }^{17-19}$ These patients with PPC had no 5-year survivors, were more likely to have recurrence in the peritoneum, and had a shorter DFS, but this did not reach statistical significance. Until more prospective data are available, only patients having had initial chemotherapy and conversion to negative washings should be considered for resection of the primary tumor.

\section{Implications of Lymph Node Location at the Time of Surgical Resection}

Surgeons who identify regional lymph node involvement at the time of surgery should proceed with curative surgical resection. Conversely, patients with nonregional lymph node metastases at the time of surgery are considered to have metastatic disease and are, therefore, not considered ideal candidates for curative surgical resection. Regional lymph nodes for cancers in the head of the pancreas are defined in the AJCC 7th Edition to be "nodes along the common bile duct, common hepatic artery (CHA), portal vein, posterior and anterior pancreaticoduodenal arcades, and along the superior mesenteric vein and right lateral wall of the superior mesenteric artery (SMA)," ${ }^{20}$ whereas the College of American Pathologists defines celiac nodes as regional lymph nodes for cancers involving the head of the pancreas. ${ }^{21}$ Regional nodes for tumors in the body or tail of the pancreas are "along the CHA, celiac axis, splenic artery, and splenic hilum." ${ }^{20}$ Therefore, this implies that aortocaval and left-sided SMA nodes are considered metastatic disease for all patients with PCA.

Data supporting these definitions are sparse. However, some literature suggests that certain lymph node basins have a poorer prognosis. For example, patients with positive CHA regional nodes have been reported as having inferior prognoses, with no patients surviving more than 4 years from resection. ${ }^{20,22,23}$ Patients with positive aortocaval lymph nodes have superior survival compared with those with CHA nodes; nonetheless, long-term survival is rare. ${ }^{24,25}$ Therefore, the authors suggest that the CHA and retropancreatic aortocaval nodal basins be sampled at the time of surgery. Rather than aborting surgery in these patients, it may be reasonable to incorporate a more aggressive adjuvant regimen and/or tailor adjuvant radiation therapy fields to encompass these nodal areas. Given the worse prognosis in this group of patients, future adjuvant therapy trials should consider stratifying patients based on "nonregional" versus "regional" lymph node metastases. Although positive lymph node metastases are a negative prognostic indicator, they do not preclude cure or long-term survival and should therefore not be considered a contraindication to an aggressive surgical approach.

\section{Management of the Primary Tumor When Metastases Are Identified at Surgery}

Previously published reports of pancreatic resection with simultaneous hepatic resections in the management of metastatic PCA reveal no survival advantage over chemotherapy alone. , $3,26,27^{\text {This sug- }}$ gests that an aggressive resectional approach to incidentally identify metastatic disease offers no survival advantage and could limit the ability to deliver systemic therapy postoperatively. If metastases are found during laparoscopic exploration, no further surgery is advised unless there is a known duodenal 
obstruction, wherein consideration should be given to a duodenal bypass (gastrojejunostomy) and cholecystectomy, because covered and uncovered biliary stents can occlude the cystic duct and lead to cholecystitis. If survival is deemed short, an endoscopically placed stent is superior. ${ }^{28}$ If metastases are discovered at open celiotomy, then prophylactic duodenal bypass and cholecystectomy with biliary bypass should be considered, because $20 \%$ of patients will eventually develop duodenal obstruction. ${ }^{29}$ It is fairly well established that an intentional R2 resection should not be attempted. ${ }^{30}$ No data exist regarding the performance of a prophylactic gastrojejunostomy at first operation versus a postoperative duodenal stent placement at the time of a later duodenal obstruction. However, a meta-analysis of duodenal stents versus gastrojejunostomy found that the latter was preferable for patients with a longer ( $>3$ months) expectation of survival. ${ }^{31}$ Most clinicians would perform nonoperative bypass procedures and reserve surgery for patients for whom endoscopic attempts failed and were candidates for chemotherapy. Intraoperative celiac plexus block might be considered if the surgeon is comfortable with the technique. ${ }^{11}$

\section{Management of Metachronous Metastatic Disease}

Most patients who undergo resection for cure will ultimately experience recurrence and die of metastatic disease. Characteristic metastatic sites are the peritoneum, liver, and lung. The value of metastasectomy has been supported in other gastrointestinal tumors and may improve outcomes in a highly selected group of patients with PCA who experience recurrence in the lung. Although not clearly understood, PCA that has a propensity to metastasize to the lung seems to have a different natural history. Investigation into the merits of pulmonary metastasectomy reveals that, in a highly selected group of patients with good performance status and with a long interval between primary resection and recurrence, pulmonary metastasectomy resulted in an improved median OS..$^{32}$ The benefits of metastasectomy for metachronous liver lesions are far less clear. Although small studies have reported improved survival benefits from resection of metachronous liver lesions, ${ }^{33}$ this practice should probably be discouraged until further investigation warrants its efficacy.
Management of the Primary Tumor in Patients With a Radiologic Complete Response of Metastatic Disease Following Chemotherapy With improved combination chemotherapy regimens, more radiologic complete responses in patients with known metastatic disease have been seen. Pathologic complete response to neoadjuvant therapy in resectable PCA is associated with a better prognosis. ${ }^{34}$ This suggests that treating patients with neoadjuvant chemotherapy with or without local therapy, such as radiation, may be important in predicting which patients are likely to benefit from resection of the primary pancreatic tumor in the future.

\section{Role of Radiation Therapy, Embolization, and Ablation in Metastatic PCA}

Although most patients with PCA die of metastatic disease, select patients may die of local progression or have primary tumors with a low burden of metastatic disease. In an autopsy study, Iacobuzio-Donahue et $\mathrm{al}^{35}$ found that $30 \%$ of patients died of localized PCA only and that these patients were more likely to have intact SMAD4/DPC4 staining seen on immunohistochemistry of their tumor. Conversely, patients with SMAD4/DPC4-mutated status were more likely to die of widespread metastatic disease. Subsequent retrospective and prospective reports have shown conflicting results regarding SMAD4 status. $^{36-38}$ Nevertheless, knowing SMAD4/DPC4 status may be helpful in determining which patients with metastatic disease may be good candidates for local radiation therapy.

Currently, in patients with metastatic PCA, standard chemoradiation is used only for local palliation or pending obstruction, because it can delay administration of full-dose chemotherapy. However, if a patient with PCA has oligometastatic disease that has been stable on chemotherapy for an extended period (eg, >6 months) or if a patient has local progression only, it may be reasonable to deliver chemoradiation during a planned break from chemotherapy.

An alternative method that may provide benefit in these patients is SBRT, a radiation technique that involves the delivery of focused radiation over 1 to 5 days to the pancreatic tumor or metastatic lesion with a small margin. ${ }^{39}$ Historically, a single treatment with SBRT (25 Gy x 1 fraction) to the pancreas has been associated with high rates $(>40 \%)$ of late grade 2 or 
greater gastrointestinal toxicity. ${ }^{40,41}$ However, preliminary data from a recent multicenter phase II trial for locally advanced PCA demonstrated that more acceptable late gastrointestinal toxicity $(<10 \%)$ may be achieved with fractionated SBRT (3-5 treatments over 1-2 weeks). ${ }^{42}$ Importantly, given the short course of therapy, SBRT may be more easily integrated with aggressive systemic chemotherapy regimens, such as FOLFIRINOX (infusional 5-FU, leucovorin, oxaliplatin, and irinotecan), gemcitabine plus nabpaclitaxel, or GTX (gemcitabine, docetaxel, and capecitabine). Furthermore, it is possible that SBRT could be used to treat locally advanced tumors in an attempt to prevent local progression, even in the setting of oligometastatic disease.

Although SBRT could be used in the treatment of metastatic pancreatic lesions, data are limited because these patients often develop multiple liver or lung metastases that are not amenable to focused SBRT. Moreover, historical systemic regimens are not able to prevent the subsequent spread of metastases. Nevertheless, multiple studies have established SBRT as an effective and well-tolerated treatment modality for colorectal liver metastases. ${ }^{43,44}$ With improved systemic therapies, SBRT may be more widely used to treat the primary tumor in select patients with a low burden of metastatic disease. SBRT can also be used to treat liver and lung metastases as long as there is an ample reserve of nonirradiated normal tissue.

In addition to SBRT, thermal ablation using microwave, cryoablation, or radiofrequency ablation (RFA) techniques has also shown promising results in treating limited metastatic PCA. ${ }^{45,46}$ Laparoscopic RFA, which has been suggested to be theoretically more precise than percutaneous RFA, appears to safely provide local control to hepatic tumors; in fact, a 16-year experience at the Cleveland Clinic recently reported on RFA as a technique to minimize morbidity and mortality. ${ }^{47}$ A nonthermal ablation technique called irreversible electroporation can also be used for focal tumor ablation in small $(<4 \mathrm{~cm})$ locally recurrent/persistent and unresectable PCA. It has been suggested that this technique may limit damage to adjacent structures and blood vessels, although efficacy and toxicity data are lacking. ${ }^{48,49}$

Intra-arterial radioembolization, although its role in metastatic PCA is unclear, has shown benefit in patients with liver metastases from breast, colon, and neuroendocrine tumors. ${ }^{50,51}$ Radioembolization with yttrium-90 microspheres is feasible in patients with large-burden metastatic PCA, ${ }^{52}$ although limited data warrant additional clinical trials to determine its effect on clinical outcomes. After a Whipple resection or a biliary bypass, caution should be used when treating metastatic lesions with surgery, ablation, or radioembolization, because there may be a higher risk of liver abscess formation due to obligate colonization of the bile ducts from the biliary-enteric anastomosis. ${ }^{53}$ This risk may be less with the use of SBRT, because this technique is noninvasive and the necrosis occurs more slowly when compared with ablation or transarterial chemoembolization. However, further investigation is necessary.

\section{Future Directions and Conclusions}

\section{Biomarkers}

Patient selection is one of the most important variables when considering therapy for advanced-stage disease, and predictive biomarkers are needed to improve patient stratification into the most appropriate treatment regimens. Biomarkers allow for individualized therapies to target specific mutations and molecular pathways that seem to be closely linked to pancreatic carcinogenesis, such as KRAS, p16/ CDKN2A, TP53, BRCA2, PALB2, and SPARC. ${ }^{54,55}$ Despite the identification of promising molecular targets, no biomarkers except for carbohydrate antigen (CA 19-9) have been implemented for routine clinical use; levels greater than $200 \mathrm{U} / \mathrm{mL}$ are highly suggestive of metastatic disease. SMAD4/DPC4 is another potential predictive marker for metastatic disease. ${ }^{35}$ Predicting patterns of locoregional versus metastatic disease progression may allow physicians to accurately determine which patients may benefit from more aggressive local therapy. More of these types of predictive biomarkers will be available in the near future as information from large-scale sequencing projects identifies key genetic drivers.

\section{Current Investigational Agents}

Although FOLFIRINOX and gemcitabine/nabpaclitaxel have demonstrated clinically significant activity in metastatic PCA, improvements in response rates and survival are still needed. Investigation of novel agents for the treatment of PCA is underway. Whether any of these approaches will prove to be beneficial is uncertain. Nevertheless, 


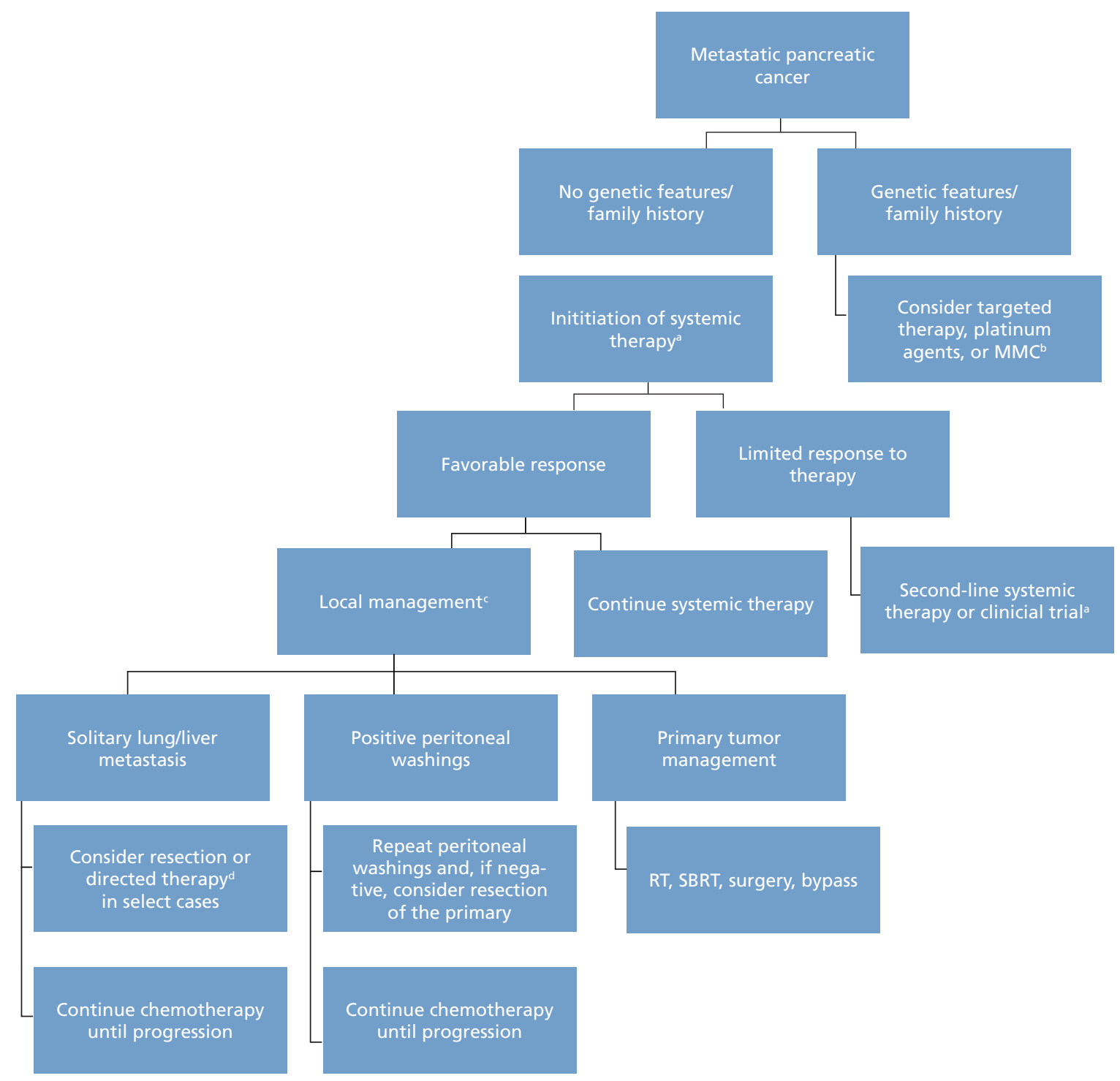

Figure 1 Treatment algorithm for patients who present with metastatic pancreatic cancer.

Abbreviations: MMC, mitomycin C; RT, radiation therapy; SBRT, stereotactic body radiation therapy.

aBased on performance status (see NCCN Guidelines for Pancreatic Adenocarcinoma ${ }^{14}$ ).

${ }^{b}$ Cui Y, Brosnan JA, Blackford AL, et al. Genetically defined subsets of human pancreatic cancer show unique in vitro chemosensitivity. Clin Cancer Res 2012; 18:6519-6530.

If bleeding, pain, or obstruction, consider palliative RT, surgery bypass, duodenal stent, and/or pain block.

dDirected therapies include SBRT and ablation (limited data to support).

higher response rates with more durable complete responses will eventually lead to long-term survival in select patients with metastatic PCA. In the meantime, integration of the other modalities described earlier, when appropriately used, is likely to improve survival and quality of life, even when cure is not possible. Moreover, efforts to develop predictive biomarkers to better select cytotoxic agents, radiation therapy, molecular therapeutics, or immunotherapy will likely expand the proportion of patients being considered for local therapies directed against the primary tumor or the metastatic compartment.

\section{Principles for Management of Patients With Metastatic PCA}

Based on review of the current literature, it is premature to speculate on the broad application of aggressive multimodality therapy for patients with metastatic disease. However, based on the current knowledge and clinical practice, it is beginning to be understood that for a handful of highly select patients, treatment of the metastatic site or primary tumor may offer meaningful clinical benefit. Some guiding principles to consider 
when determining treatment of these select cases are as follows (Figure 1):

- Patients with good performance status and limited metastatic disease should be referred to a high-volume PCA center to:

- Be considered for enrollment in a clinical trial, and

- Undergo multidisciplinary review of other potential interventions beyond systemic therapy, intended to control limited metastatic disease and/or the primary tumor with careful attention to sequencing.

- Patients should be initially treated with a minimum of 4 to 6 months (based on tolerability and response) of systemic chemotherapy to identify who may benefit from aggressive local management or control of limited residual metastatic disease.

- If prolonged control of metastases is established with systemic therapy, select patients should be considered for minimally invasive interventions to address limited residual metastatic disease, particularly of the lung.

- Patients who achieve a complete response of metastatic disease after chemotherapy:

- Should be considered for surgical resection of the primary only after a 2- to 3-month break from full-dose systemic therapy. If indicated, chemoradiation or SBRT to the primary could be considered during this period.

- In patients with known prior metastatic disease, laparoscopic staging with peritoneal washings should be performed and the patient should have negative cytology before a resection of the primary tumor or metastatic site is attempted.

\section{Summary}

New combinations of systemic therapy are sufficiently active to now consider more aggressive multimodal therapy for select patients with limited metastatic disease. Furthermore, surgical removal of the primary tumor or metastatic site may now be an appropriate strategy for a small subset of patients experiencing a complete radiographic response to systemic therapy. However, more studies are needed. Such interven- tions are best performed after multidisciplinary review in high-volume centers with expertise in newer radiation techniques, minimally invasive surgery, and interventional radiology.

\section{Acknowledgments}

The authors would like to thank Lauren M. Rosati, BS, for her technical assistance and contribution to the revision of this manuscript.

\section{References}

1. Pisters PW, Lee JE, Vauthey JN, et al. Laparoscopy in the staging of pancreatic cancer. Br J Surg 2001;88:325-337.

2. Satoi S, Yanagimoto $H$, Toyokawa $H$, et al. Selective use of staging laparoscopy based on carbohydrate antigen 19-9 level and tumor size in patients with radiographically defined potentially or borderline resectable pancreatic cancer. Pancreas 2011;40:426-432.

3. Burris HA3rd, Moore MJ, Andersen J, et al. Improvements in survival and clinical benefit with gemcitabine as first-line therapy for patients with advanced pancreas cancer: a randomized trial. J Clin Oncol 1997;15:24032413.

4. Royal RE, Wolff RA, Crane CH. Pancreas cancer. In: Devita VT, Hellman S, Rosenberg S, eds. Cancer: Principles and Practice of Oncology. 9th Edition. Philadelphia, PA: Lippincott, Williams and Wilkins; 2011:982983.

5. Conroy T, Desseigne F, Ychou M, et al. FOLFIRINOX versus gemcitabine for metastatic pancreatic cancer. N Engl J Med 2011;364:1817-1825.

6. Von Hoff DD, Ervin T, Arena FP, et al. Increased survival in pancreatic cancer with nab-paclitaxel plus gemcitabine. N Engl J Med 2013;36:16911703.

7. Fine RL, Fogelman DR, Schreibman SM, et al. The gemcitabine, docetaxel, and capecitabine (GTX) regimen for metastatic pancreatic cancer: a retrospective analysis. Cancer Chemother Pharmacol 2008;61:167-175.

8. Arnaoutakis GJ, Rangachari D, Laheru DA, et al. Pulmonary resection for isolated pancreatic adenocarcinoma metastasis: an analysis of outcomes and survival. J Gastrointest Surg 2011;15:1611-1617.

9. Shrikhande SV, Kleeff J, Reiser C, et al. Pancreatic resection for M1 pancreatic ductal adenocarcinoma. Ann Surg Oncol 2007;14:118-127.

10. McKenzie S, Mailey B, Artinyan A, et al. The incidence and outcomes of pancreatectomy in patients with metastatic pancreatic adenocarcinoma. JOP 2010;11:341-347.

11. Lillemoe KD, Cameron JL, Kaufman HS, et al. Chemical splanchnicectomy in patients with unresectable pancreatic cancer. A prospective randomized trial. Ann Surg 1993;217:447-455; discussion 456-457.

12. Yeo CJ, Cameron JL, Sohn TA, et al. Six hundred fifty consecutive pancreaticoduodenectomies in the 1990s: pathology, complications, and outcomes. Ann Surg 1997;226:248-257; discussion 257-260.

13. Gleisner AL, Assumpcao L, Cameron JL, et al. Is resection of periampullary or pancreatic adenocarcinoma with synchronous hepatic metastasis justified? Cancer 2007;110:2484-2492.

14. Tempero MA, Arnoletti JP, Behrman S, et al. Pancreatic adenocarcinoma. J Natl Compr Canc Netw 2010;8:972-1017.

15. Warshaw AL. Implications of peritoneal cytology for staging of early pancreatic cancer. Am J Surg 1991;161:26-29; discussion 29-30.

16. Ferrone CR, Haas B, Tang L, et al. The influence of positive peritoneal cytology on survival in patients with pancreatic adenocarcinoma. J Gastrointest Surg 2006;10:1347-1353.

17. Yachida S, Fukushima N, Sakamoto M, et al. Implications of peritoneal washing cytology in patients with potentially resectable pancreatic cancer. Br J Surg 2002;89:573-578.

18. Yoshioka R, Saiura A, Koga R, et al. The implications of positive peritoneal lavage cytology in potentially resectable pancreatic cancer. World J Surg 2012;36:2187-2191. 
Herman et al

19. Meszoely IM, Lee JS, Watson JC, et al. Peritoneal cytology in patients with potentially resectable adenocarcinoma of the pancreas. Am Surg 2004; 70:208-213; discussion 213-214

20. Edge S, Byrd DR, Compton CC, et al, eds. AJCC Cancer Staging Manual. 7th Edition. New York, NY: Springer; 2010.

21. Pancreas (endocrine) cancer protocol. College of American Pathologists Web site. Available at: http://www.cap.org/apps/docs/cancer_ protocols/2005/pancreasendo05_pw.pdf. Accessed January 6, 2014.

22. Cordera F, Arciero CA, Li T, et al. Significance of common hepatic artery lymph node metastases during pancreaticoduodenectomy for pancreatic head adenocarcinoma. Ann Surg Oncol 2007;14:2330-2336.

23. LaFemina J, Chou JF, Gonen M, et al. Hepatic arterial nodal metastases in pancreatic cancer: Is this the node of importance? J Gastrointest Surg 2013;17:1092-1097.

24. Shimada K, Sakamoto Y, Sano T, Kosuge T. The role of paraaortic lymph node involvement on early recurrence and survival after macroscopic curative resection with extended lymphadenectomy for pancreatic carcinoma. J Am Coll Surg 2006;203:345-352.

25. Masui T, Kubota T, Aoki K, et al. Long-term survival after resection of pancreatic ductal adenocarcinoma with para-aortic lymph node metastasis: case report. World J Surg Oncol 2013;11:195.

26. Seelig SK, Burkert B, Chromik AM, et al. Pancreatic resections for advanced M1-pancreatic carcinoma: the value of synchronous metastasectomy. HPB Surg 2010;2010:579672.

27. Takada T, Yasuda H, Amano H, et al. Simultaneous hepatic resection with pancreato-duodenectomy for metastatic pancreatic head carcinoma: does it improve survival? Hepatogastroenterology 1997;44:567-573.

28. Artifon EL, Sakai P, Cunha JE, et al. Surgery or endoscopy for palliation of biliary obstruction due to metastatic pancreatic cancer. Am J Gastroenterol 2006;101:2031-2037.

29. Lillemoe $\mathrm{KD}$, Cameron JL, Hardacre JM, et al. Is prophylactic gastrojejunostomy indicated for unresectable periampullary cancer? A prospective randomized trial. Ann Surg 1999;230:322-328; discussion 328-330.

30. Tachezy M, Bockhorn M, Gebauer F, et al. Bypass surgery versus intentionally incomplete resection in palliation of pancreatic cancer: is resection the lesser evil? J Gastrointest Surg 2011;15:829-835.

31. Jeurnink SM, van Eijck CH, Steyerberg EW, et al. Stent versus gastrojejunostomy for the palliation of gastric outlet obstruction: a systematic review. BMC Gastroenterol 2007;7:18.

32. Poruk KE, Kim Y, Cameron JL, et al. What is the significance of indeterminate pulmonary nodules in patients undergoing resection for pancreatic adenocarcinoma? J Gastrointest Surg 2015;19:841-847.

33. Klempnauer J, Ridder GJ, Piso P, Pichlmayr R. Is liver resection in metastases of exocrine pancreatic carcinoma justified? Chirurg 1996;67:366-370.

34. Zhao Q, Rashid A, Gong Y, et al. Pathologic complete response to neoadjuvant therapy in patients with pancreatic ductal adenocarcinoma is associated with a better prognosis. Ann Diagn Pathol 2012;16:29-37.

35. Iacobuzio-Donahue CA, Fu B, Yachida S, et al. DPC4 gene status of the primary carcinoma correlates with patterns of failure in patients with pancreatic cancer. J Clin Oncol 2009;27:1806-1813.

36. Winter JM, Tang LH, Klimstra DS, et al. Failure patterns in resected pancreas adenocarcinoma: lack of predicted benefit to SMAD4 expression. Ann Surg 2013;258:331-335.
37. Blackford A, Serrano OK, Wolfgang CL, et al. SMAD4 gene mutations are associated with poor prognosis in pancreatic cancer. Clin Cancer Res 2009; 15:4674-4679.

38. Herman JM, Fan KY, Wild AT, et al. Correlation of Smad4 status with outcomes in patients receiving erlotinib combined with adjuvant chemoradiation and chemotherapy after resection for pancreatic adenocarcinoma. Int J Radiat Oncol Biol Phys 2013;87:458-459.

39. Chang BK, Timmerman RD. Stereotactic body radiation therapy: a comprehensive review. Am J Clin Oncol 2007;30:637-644.

40. Hoyer M, Roed H, Sengelov L, et al. Phase-II study on stereotactic radiotherapy of locally advanced pancreatic carcinoma. Radiother Oncol 2005;76:48-53

41. Schellenberg D, Goodman KA, Lee F, et al. Gemcitabine chemotherapy and single-fraction stereotactic body radiotherapy for locally advanced pancreatic cancer. Int J Radiat Oncol Biol Phys 2008;72:678-686.

42. Herman JM, Chang DT, Goodman KA, et al. Phase 2 multi-institutional trial evaluating gemcitabine and stereotactic body radiotherapy for patients with locally advanced unresectable pancreatic adenocarcinoma. Cancer 2015;121:1128-1137.

43. Chang DT, Swaminath A, Kozak M, et al. Stereotactic body radiotherapy for colorectal liver metastases: a pooled analysis. Cancer 2011;117:40604069 .

44. Dawood O, Mahadevan A, Goodman KA. Stereotactic body radiation therapy for liver metastases. Eur J Cancer 2009;45:2947-2959.

45. Brown DB, Narayanan G. Interventional radiology and the pancreatic cancer patient. Cancer J 2012;18:591-601.

46. Park JB, Kim YH, Kim J, et al. Radiofrequency ablation of liver metastasis in patients with locally controlled pancreatic ductal adenocarcinoma. J Vasc Interv Radiol 2012;23:635-641.

47. Birsen O, Aliyev S, Aksoy E, et al. A critical analysis of postoperative morbidity and mortality after laparoscopic radiofrequency ablation of liver tumors. Ann Surg Oncol 2014;21:1834-1840.

48. Weiss MJ, Wolfgang CL. Irreversible electroporation: a novel pancreatic cancer therapy. Curr Probl Cancer 2013;37:262-265.

49. Al-Sakere B, Andre F, Bernat C, et al. Tumor ablation with irreversible electroporation. PLoS One 2007;2:e1135.

50. Ho AS, Picus J, Darcy MD, et al. Long-term outcome after chemoembolization and embolization of hepatic metastatic lesions from neuroendocrine tumors. AJR Am J Roentgenol 2007;188:1201-1207.

51. Stuart JE, Tan B, Myerson RJ, et al. Salvage radioembolization of liverdominant metastases with a resin-based microsphere: initial outcomes. J Vasc Interv Radiol 2008;19:1427-1433.

52. Cao C, Yan TD, Morris DL, Bester L. Radioembolization with yttrium-90 microspheres for pancreatic cancer liver metastases: results from a pilot study. Tumori 2010;96:955-958.

53. De Jong MC, Farnell MB, Sclabas G, et al. Liver-directed therapy for hepatic metastases in patients undergoing pancreaticoduodenectomy: a dual-center analysis. Ann Surg 20101;252:142-148.

54. Jazieh KA, Foote MB, Diaz LA Jr. The clinical utility of biomarkers in the management of pancreatic adenocarcinoma. Semin Radiat Oncol 2014;24:67-76.

55. Wolfgang CL, Herman JM, Laheru DA, et al. Recent progress in pancreatic cancer. CA Cancer J Clin 2013;63:318-348. 